\title{
Zur Taxonomie der Gattung Clypeocampulum GAULD (Hymenoptera, Ichneumonidae)
}

\author{
Mit 18 Figuren \\ HEINZ SCHNEE ${ }^{1}$ \\ ${ }^{1}$ Birkenweg 18, 04416 Markkleeberg, Deutschland. - Heinz.Schnee@web.de \\ Published on 2014-07-31
}

\section{Summary}

The genus Clypeocampulum Gauld, 1976 is revised. The species name of the type species C. tibiale GaUld, 1976 is a new synonym of C. lubricum (AtAnAsov, 1975), comb. $\mathrm{n}$. The validity of the genus is confirmed by new features. Clypeocampulum barbarae sp. n. from Spain and Clypeocampulum gauldi sp. n. from Turkey are described. A key enables the determination of the species. The distribution area of C. lubricum reaches from East Mediterranean countries to Central Asia. Some species of Pieridae (Lepidoptera) are recorded as hosts of C. barbarae and C. lubricum.

\section{Zusammenfassung}

Die Gattung Clypeocampulum Gauld, 1976 wird revidiert. Ihre typische Art ist C. tibiale Gauld, 1976. Dieser Name ist ein neues Synonym von C. lubricum (Atanasov, 1975), comb. n. Die Eigenständigkeit des Genus wird an Hand neuer Merkmale bestätigt. Es werden Clypeocampulum barbarae sp. n. aus Spanien und Clypeocampulum gauldi sp. n. aus der Türkei beschrieben. Ein Bestimmungsschlüssel ermöglicht die Determination der Arten. Bei C. lubricum reicht das Areal vom östlichen Mittelmeerraum bis nach Mittelasien. Als Wirte von C. barbarae und C. lubricum dienen einige Arten aus der Lepidopteren-Familie Pieridae.

\section{Keywords}

Ichneumonidae, Anomaloninae, Clypeocampulum, new species, Pieridae as hosts

\section{Einleitung}

Die Gattung Clypeocampulum gehört zur Unterfamilie Anomaloninae. Sie wurde von Gauld (1976) im Rahmen seiner „...Classification of the Anomaloninae..." aufgestellt. Er schloss in sie als typische Art Clypeocampulum tibiale GAuld, 1976 aus der Türkei ein. Ihm lagen nur der Holotypus und ein Paratypus dieser Art vor, beide männlichen Geschlechts. Folgende
Merkmale verwendete er vor allem zur Trennung seiner Gattung von allen übrigen paläarktischen Genera der Unterfamilie:

- Clypeus grob längsrunzlig skulptiert

- Occipitalleiste undeutlich

- Mitteltibien mit nur je einem Endsporn. 
Die Untersuchung eines umfangreicheren Materials hat ergeben, dass diese Merkmale keineswegs immer zutreffen und deshalb die Gattung nicht hinreichend kennzeichnen.

In diesem Beitrag wird GAulds Beschreibung ergänzt. Außerdem werden zwei neue Arten beschrieben und es wird die verwandtschaftliche Stellung der Gattung diskutiert.

\section{Material und Methoden}

Die hier verwendete Terminologie des Flügelgeäders folgt dem Rohwer \& Gahan-System nach Townes (1971). Dagegen hat sich GAuld (1976) am Comstock \& NeEDHAM -System orientiert. Die untersuchten Tiere stammen aus folgenden Sammlungen:

BME Bohart Museum of Entomology Davis, USA

HNHM Hungarian Natural History Museum Budapest, Ungarn

BMNH Museum of Natural History London, Großbritanien

NMS National Museums of Scotland Edinburgh, Großbritanien

OLML Oberösterreichisches Landesmuseum Linz, Österreich

ZIN Zoological Institute of Russian Academy of Sciences St. Petersburg, Rußland

RMNH Rijksmuseum van Natuurlijke Historie Leiden, Niederlande

Schnee Coll. Heinz Schnee, Markkleeberg, Deutschland

Die digitalen Fotografien wurden mit der Kamera JVC KYF-F75U am Leica Z6 APO Zoomsystem aufgenommen. Die Verrechnung der Bildstapel erfolgte mit Combine ZP.

\section{Ergänzung und Korrektur der Gattungsbe- schreibung von Clypeocampulum durch GAULD (1976: $42 \mathrm{ff}$ )}

Fühler höchstens halb so lang wie der Körper; Clypeus apikal stumpfwinklig oder rundlich vorgezogen, ohne Mittelzahn, grob und dicht bis zum Teil furchig punktiert; Mandibeln einzähnig oder zweizähnig mit nur sehr kleinem unteren Zahn und Tendenz zur Einzähnigkeit; Occipitalleiste vorhanden, bei C.lubricum aber oben hinter den Augen undeutlich oder erloschen; Schläfen im Profil nach oben deutlich verengt.

Praepectalleiste sowohl ventral als auch lateral markant und den Vorderrand der Mesopleuren erreichend; kurze Sternauli vorhanden; Notauli fehlend oder schwach angedeutet; große stumpfe Metasternalhöcker vorhanden.
Mitteltibien in der Regel mit je zwei apikalen Spornen, bei C. lubricum eine Tendenz zur völligen Reduktion des inneren Sporns; Hintertarsen in beiden Geschlechtern deutlich verdickt.

Im Vorderflügel Subdiscoideus in der Mitte des stark geknickten Postnervulus entspringend, Nervus recurrens postfurcal; Discoidella im Hinterflügel etwas unter bis etwas über der Mitte des Nervellus entspringend.

Aedoeagus (in Lateralsicht) am apikalen Ende stark konkav eingebuchtet, proximales Ende einfach, nicht erweitert.

In Gaulds (1976) Bestimmungsschlüssel für die Gattungen und Untergattungen der Anomaloninae muss Clypeocampulum an zwei Stellen eingeordnet werden, da zwei der hier behandelten Arten zwei Mitteltibiensporne haben und bei der dritten Art die Zahl dieser Sporne zwischen 1 und 2 schwankt.

3 (1) Mid tibia with two distinct apical spurs 4

Mid tibia as a rule with a single apical spur. ........... 41

9 (6) Apex of clypeus concave or truncate, without a median apical tooth (Text-fig. 159 ${ }^{1}$ ); mesoscutum in profile smoothly and evenly rounded, notauli absent; without metasternal humps (Mediterranean and further Palaearctic region). .. $9 \mathrm{a}$

- Apex of clypeus obtuse angular or convex, without a median apical tooth; mesoscutum in profile evenly rounded, notauli absent or at most weakly indicated; with great obtuse metasternal humps (Mediterranean). Clypeocampulum (partim)

- Apex of clypeus convex or pointed, usually with a median apical tooth, if rarely this tooth is absent then either mesoscutum in profile is abruptly rounded, or notauli are present, if rarely clypeus is truncate or weakly concave then median apical tooth is distinct (different zoogeographical regions). 10

45 (43) Clypeus elaborately sculptured (Text-fig. 167'); mandible bidentate, rarely with one developed tooth; mid tibia mostly with one apical spur, but in some specimens with two spurs; tarsal claws small, basally pectinate; occipital carina centrally interrupted by a series of discontinuities (Mediterranean to Eastern Palaearctic). Clypeocampulum (partim)

- Clypeus smooth; mandible unidentate; mid tibia always with one apical spur; tarsal claws long, weakly curved, not pectinate; occipital carina complete. (Western Nearctic). Liopterna Townes 


\section{Bestimmungsschlüssel für die Arten der Gattung Clypeocampulum}

1. Mesoscutum mit schwachen Notauli (Fig. 1); Mesopleuren sehr dicht runzlig und oben streifig punktiert; Clypeus apikal abgerundet; Gesicht und Clypeus ganz gelb (Fig. 2); Hinterbeine (außer Coxen und Trochanteren) rot (Fig. 3). C. gauldi sp. n.

Mesoscutum ohne Notauli (Fig. 5 und 10); Mesopleuren mehr oder weniger dicht, aber nicht runzlig punktiert; Clypeus apikal leicht stumpfwinklig vorgezogen; Gesicht und Clypeus ganz oder überwiegend schwarz (Fig. 6, 11, 12); Hinterbeine schwarz, mitunter aber mit rotbraunen Aufhellungen der Hinterfemora (Fig. 8 und 16). ......... 2

2. Genalleiste im unteren Drittel der Schläfen ab einem leichten Knick gerade bis leicht konkav auf die Mandibelbasis treffend (Fig. 7); Mandibeln einzähnig; Kopf hinter den Augen verengt, Occipitalleiste markant (Fig. 5); Epomia deutlich; Praepectalleiste ventral hinter den Vordercoxen lamellenartig erhöht; Hintercoxen fein und zerstreut punktiert; Gesicht und Clypeus ganz schwarz (Fig. 6); Clasper (= Gonoforceps) am Ende konvex. .... C. barbarae sp. n.

Genalleiste leicht konvex auf die untere Mandibelecke treffend, ohne Knick (Fig. 17); Mandibeln meist zweizähnig, mit sehr kleinem unteren Zahn; Kopf hinter den Augen nicht verengt und Occipitalleiste dort undeutlich (Fig. 10); Epomia nur angedeutet; Praepectalleiste ventral hinter den Vordercoxen nicht erhöht; Hintercoxen vor allem dorsal grob und dicht punktiert; Gesicht und Clypeus schwarz mit gelben inneren Orbiten (Fig. 11 und 12), mitunter mit gelbem Mittelstreifen; Clasper apikal mehr oder weniger konkav (Fig. 15). C. lubricum (AtANASOv)

Key to the species of the genus Clypeocampulum

1. Mesoscutum with weak notauli (Fig. 1); mesopleura very densely wrinkled and above streaky punctate; clypeus apically rounded; face and clypeus completely yellow (Fig. 2); hind legs (except coxae and trochanters) red (Fig. 3). C. gauldi sp. n.

Mesoscutum without notauli (Figs 5 and 10); mesopleura more or less densely but not wrinkled punctate; clypeus apically obtuse - angled protruding; face and clypeus completely or predominantly black; hind legs black or with reddish-brown hind femora (Figs 8 and 16).

2. Genal carina on lower third of temple with weak bend, between bend and base of mandible straight to weakly concave (Fig. 7); mandibles unidentate; head contracting behind the eyes, occipital carina distinct (Fig. 5); epomia present; prepectal carina behind fore coxae raised lamellar; hind coxae finely and scarcely punctate; face and clypeus completely black (Fig. 6); clasper (= gonoforceps) apically convex. C. barbarae sp. n.

Genal carina joining base of mandible in a weakly convex line, without a bend (Fig. 17); mandibles mostly bidentate, but lower tooth very small; head behind eyes not contracted and occipital carina indistinct (Fig. 10); epomia only indicated; prepectal carina behind fore coxae not raised; hind coxae particularly dorsally with coarse and dense punctures; face and clypeus black with yellow inner orbits (Figs 11 and 12), sometimes with a yellow median streak; clasper apically more or less concave (Fig. 15). C. lubricum (Atanasov)

\section{Artbeschreibungen}

\section{Clypeocampulum barbarae sp. n.}

(Fig. 5-9)

Holotypus: + : „Spain: Barcelona Valles Oriental, El Puig, 1026 m Montseny Anthocharis euphenoides 10.6.96 Biscutella laevigata em. 18.5.97 C. Stephanescu", "Erigorgus". (Wirtspuppe vorhanden) (NMS).

Paratypen: 1 1 : „Spain: Barcelona Valles Oriental, El Puig, 1026 m UTM 452: 4627 C. Stephanescu Anthocharis euphenoides 8.6.05. pup. 14.6.05, em. 12.5.2006";

2 우 우: dito, em. 22.5.2006;

2 우 우: dito, coll. 6.05. pup. 15.6.05, em. 15.5.2006;

$1 \mathrm{o}^{\star}$ : dito, 6.5.05, pup. 19.6.05, em. 15.5.2006, coll in penult. instar;

1 ㅇ: "Spain: Barcelona Bages coll d'Estenalles Sant LLorenc del Munt, $900 \mathrm{~m}$. Attacking $4^{\text {th }}$ instar Anthocharis euphenoides, Biscutella laevigata 30.5.1997 C. Stephanescu" (alle NMS);

1 ㅇ: „Spain: Barcelona Valles Oriental, El Puig, $1026 \mathrm{~m}$ UTM 452: 4627 C. Stephanescu Anthocharis euphenoides 8.6.05. pup. 14.6.2006" (Schnee);

1 o : "Hispania Cuenca (Korb)" (HNHM);

1 ㅇ: "Spanien: Almeria Bayarcal 24.6.1988 leg. M. Schwarz" (Schnee);

1 ㅇ: „E, Prov. Granada Sa. Nevada, 1400 m, östl. Portugos 10.6.1991 leg. Tiefenthaler“(OLML).

Holotypus: Körper $13 \mathrm{~mm}$, Vorderflügel $7 \mathrm{~mm}$, Fühler $6 \mathrm{~mm}$ lang (Habitus-Fig. 8).

Kopf: Innere Augenränder nach unten schwach konvergierend (Fig. 6), Gesicht uneben, dicht punktiert, aber dennoch mit Glanz; Clypeus vom Gesicht etwas erhaben 
abgesetzt, unterschiedlich grob punktiert, stellenweise furchig, Endrand schwach stumpfwinklig vorgezogen; Wangenlänge 0,5 der basalen Mandibelbreite, Mandibeln einzähnig; Schläfen im unteren Drittel verengt, da Genalleiste mit leichtem Knick und dann gerade bis leicht konkav zur Mandibelbasis verlaufend (Fig. 7), dadurch Oralleiste in Lateralsicht deutlich sichtbar, Schläfen oben dichter, unten zerstreuter punktiert; Stirn mit deutlichem Mittelkiel, runzlig bis nadelrissig; Occipitalleiste hinter Ocellen schwächer, aber nicht unterbrochen; Kopf hinter den Augen etwas verengt (Fig. 5); 34 Fühlerglieder, 1. Geißelglied etwas länger oder so lang wie 2 . und 3. zusammen, 3,0-mal so lang wie breit und 1,9-mal so lang wie 2 . Glied, mittlere Glieder quadratisch.

Mesosoma: Pronotum in unterer Hälfte grob runzlig, oben zerstreut punktiert und mit etwas Glanz, Epomia vorhanden; Mesopleuren grob punktiert mit glänzenden Zwischenräumen, Speculum fehlt, Praepectalleiste hinter Vordercoxen lamellenartig erhöht, seitlich scharf bei 0,4 des hinteren Pronotumrandes endend, Mesosulcus wenig vertieft; Mesoscutum glänzend, da fein und zerstreut punktiert, Notauli fehlend; Scutellum gewölbt, zerstreut punktiert und glänzend, ohne Seitenleisten; Propodeum mitten etwas längs eingedellt, grob netzrunzlig bis unregelmäßig runzlig, bis 0,4 der Hintercoxenlänge reichend.

Beine: Coxen glänzend, sehr zerstreut punktiert; Mitteltibien apikal mit je zwei Spornen, der innere Sporn 0,7-mal so lang wie der äußere; hintere Femora 5,8-mal so lang wie breit, Hintertarsen verdickt, Klauen klein, basal fein gekämmt.

Flügel: Nervulus 0,2 seiner Länge postfurcal, Subdiscoideus in der Mitte des stark geknickten Postnervulus entspringend, Nervus recurrens 1,1 der Länge des Intercubitus postfurcal; Discoidella entspringt etwas unter der Mitte des Nervellus (Fig. 9).

Metasoma: Spirakeln des 1 . Tergits in 0,75 seiner Länge gelegen, Postpetiolus nach hinten etwas verbreitert, Bohrerklappen 0,7-mal so lang wie Hinterbasitarsen.

Färbung: Schwarz; Scheitel beiderseits oben mit undeutlichen dunkelrotbraunen Flecken, Vorder- und Mittelbeine (außer Coxen) dunkelrotbraun, Postpetiolus und Tergite 2-4 rotbraun (2. Tergit oben schwarz), Flügelgeäder einschließlich Stigma dunkelbraun; Körperbehaarung weiß.

Männchen: Innere Augenränder schwächer nach unten konvergierend; Clasper apikal konvex; Aedoeagus in Form und Mikroskulptur dem von C.lubricum sehr ähnlich (cf. Fig. 18b).

Variabilität: Schwankungsbreite der Körpermaße: Körperlänge $12-15 \mathrm{~mm}$, Vorderflügel $6,0-7,5 \mathrm{~mm}$,
Fühler 5,0-6,5 mm; 32 bis 34 Fühlerglieder; winziges Rudiment des unteren Mandibelzahns selten vorhanden (Paratypus aus Budapest); innerer Sporn der Mitteltibien 0,3 bis 0,7 -mal so lang wie äußerer; hintere Femora 5,5-6,5-mal so lang wie breit; Nervus recurrens $0,2-1,1$ der Länge des Intercubitus postfurcal.

Derivatio nominis: Die neue Art wird nach dem Vornamen meiner Frau benannt.

Biologie: Wie den Funddaten entnommen werden kann, ist die Art von Stephanescu in Spanien aus Anthocharis euphenoides Staudinger, 1869 (Lepidoptera, Pieridae) gezüchtet worden.

\section{Clypeocampulum gauldi sp. $\mathrm{n}$. \\ (Fig. 1-4)}

Holotypus: ơ: „TURKEY Ayas Dagi 3800feet (AnkaraBaypazari Rd.) 30.6.1959 K. M. Guichard“ (BMNH).

Körper 13 mm, Vorderflügel 6,8 mm, Fühler 6,5 mm lang (Habitus-Fig. 3).

Kopf: Innere Augenränder fast parallel, Gesicht mitten etwas zerstreuter, seitlich dichter punktiert; Clypeus sehr grob runzlig punktiert, apikal etwas konvex vorgezogen, Endrand transparent; Wangenlänge 0,45 der basalen Mandibelbreite, Mandibeln einzähnig (mit winzigem Rudiment des unteren Endzahns); Schläfen unter der Mitte am Breitesten, nach oben stark verschmälert, oben dichter und feiner, unten mit Punktdurchmesser-Abstand punktiert; Occipitalleiste vollständig; Kopf hinter den Augen konvex erweitert, Stirnkiel stark entwickelt, Stirn und Scheitel grob runzlig; 35 Fühlerglieder, 1. Geißelglied kürzer als 2. und 3. zusammen und 3,7-mal so lang wie breit, 2. Geißelglied doppelt so lang wie breit, mittlere Glieder etwas länger als breit.

Mesosoma: Pronotum schräg nach oben runzelstreifig, ganz oben dicht punktiert, Epomia und Speculum undeutlich; Mesopleuren sehr dicht runzlig, oben streifig punktiert, Praepectalleiste in ganzer Länge scharf, hinter Vordercoxen nicht lamellenartig; Mesoscutum fein und relativ dicht punktiert, dennoch glänzend, flache Notauli vorhanden, vor Scutellum querrunzelstreifig, Scutellum wenig gewölbt, nach hinten flach abfallend, mit deutlichen basalen Seitenleisten; Propodeum und Metapleuren grob netzrunzlig, bis 0,4 der Länge der Hintercoxen reichend.

Beine: Linke Mitteltibia mit 2 Endspornen (rechte Mitteltibia fehlt); Hintercoxen oben sehr dicht, unten zerstreuter punktiert, Hintertrochanteren dick, Trochantellen unten abgeflacht, Hinterfemora 4,6-mal so lang wie breit, Hintertarsen mit den Gliedern 1-3 verdickt, Klauen basal etwas gekämmt. 

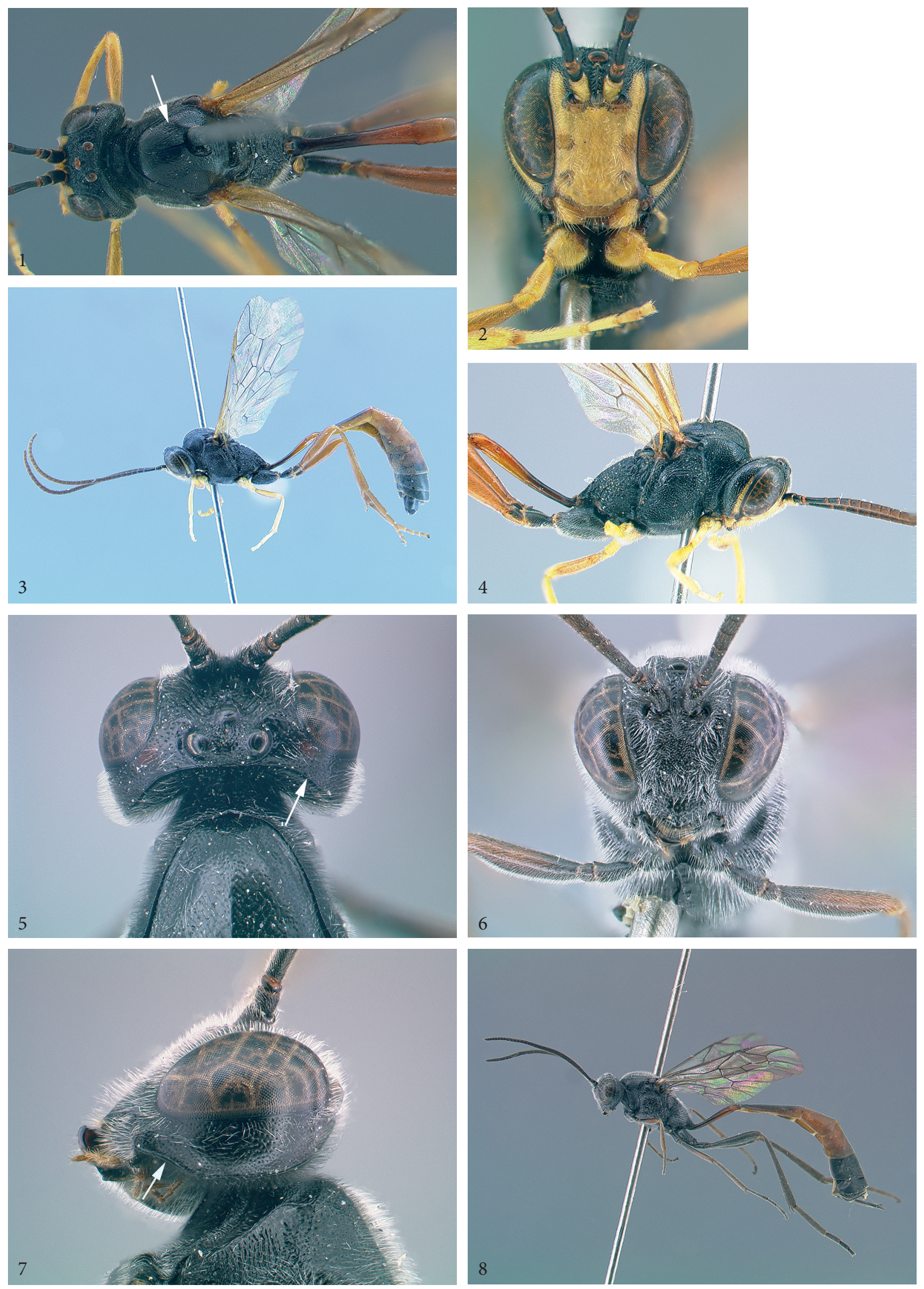

Fig. 1-4: $\sigma^{\top}$ Holotypus Clypeocampulum gauldi sp. n.; 1 Kopf und Mesosoma in Dorsalsicht, 2 Kopf von vorn, 3 Habitus lateral, 4 Kopf und Mesosoma lateral.

Fig. 5-8: ㅇ Paratypus Clypeocampulum barbarae sp. n.; 5 Kopf und Mesoscutum dorsal, 6 Kopf von vorn, 7 Kopf lateral, 8 Habitus lateral. 
Flügel: Nervulus um Aderstärke postfurcal, Subdiscoideus in 0,56 der Länge des Postnervulus entspringend, Nervus recurrens um 0,5 der Intercubitus-Länge postfurcal, Discoidella entspringt in 0,67 der Länge des Nervellus.

Metasoma: Spirakeln des 1 . Tergits in 0,76 seiner Länge gelegen, 1. Sternit in 0,5 der Postpetioluslänge endend, Clasper am Ende abgerundet.

Färbung: Schwarz; Gesicht und Clypeus, Scapus unten, innere Orbiten bis in Höhe der Vorderocellen, äußere Orbiten in den unteren zwei Dritteln ihrer Länge, Mandibeln zum größten Teil, Vorder-und Mittelbeine einschließlich Coxen ventral gelb; Hinterbeine (außer Coxen und teilweise Trochanteren), Metasoma (außer Petiolus in der Basalhälfte, 2. Tergit dorsal in der Apikalhälfte, Tergite 5-8 und Clasper) rot; Fühlergeißel basal schwarzbraun, nach apikal rötlichbraun werdend; Flügeladern braun, Behaarung des Körpers weiß.

Derivatio nominis: Der Artname wurde zu Ehren des Entdeckers der Gattung Clypeocampulum I. D. GAULD gewählt.

Biologie: unbekannt.

Clypeocampulum lubricum (ATANASOV, 1975), comb. nov. (Fig. 10-18b)

Clypeocampulum tibiale GAULD, 1976, syn. nov.

Paratypen: 1 ㅇ, $10^{*}$ : "g. Kokshetau bl. r. Tersakkan W Akmol. Tobias 5.VI.1957”, „Paratypus Erigorgus lubricus Atanasov";

$1 \sigma^{\star}$ : „Talysh 9.VI.1967 Kasparyan“, „Paratypus Erigorgus lubricus Atanasov";

$10^{\star}$ : „pr. bereg Syrdari z. pesk. Alkakolkam 1.-11.5.1968“ (alle RASZIN).

Holotypus: $\sigma^{\star}$ : „TURKEY: Mugla $40 \mathrm{kms}$ Fethiye-Ortaca Rd. 15.IV.1962", "Clypeocampulum tibiale Gauld det. I. D. Gauld 1974“(BMNH).

\section{Weitere Exemplare:}

Griechenland: $2 \sigma^{\star} \sigma^{*}$ : Greece Delphi 9.5.1971 J. v. d. Vecht (RMNH);

$1 \sigma^{*}$ : GR/Peloponnes, Vrachneika 28.4.2001, leg. H. Schnee; 3 ㅇ ㅇ : GR/Platania, $38 \mathrm{~km}$ SE Volos $39^{\circ} 09.43$

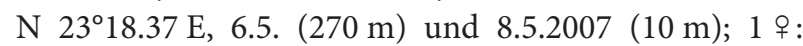
GR/Exochori/21 km SE Kalamata $36^{\circ} 54.47 \mathrm{~N} 22^{\circ} 16.67 \mathrm{E}$, $400 \mathrm{~m}, 30.4 .2007 ; 1 \mathrm{o}^{\star}$ : GR/Vorio /15 km SE Kalamata $36^{\circ} 57.59 \mathrm{~N} 22^{\circ} 14.77 \mathrm{E}, 650 \mathrm{~m}, 1.5 .2007 ; 1 \mathrm{o}^{\top}$ : GR/ Lafkos/ $33 \mathrm{~km}$ SE Volos $39^{\circ} 10.55 \mathrm{~N} 23^{\circ} 16.07 \mathrm{E}, 320 \mathrm{~m}, 8.5 .2007$; 1 \%: GR/Kambos/15 km SE Kalamata $36^{\circ} 58.00 \mathrm{~N}$ $22^{\circ} 15.50 \mathrm{E}, 750 \mathrm{~m}, 2.5 .2007$, leg. Blank, Jacobs, Schnee, Taeger (alle Schnee);

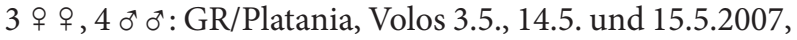
2.5., 9.5. und 14.5.2009, 8.5.2011, alle leg. K. Standfuss (Schnee);

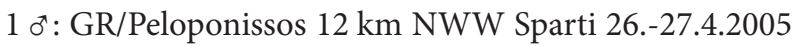

J. Halada;

$1 \mathrm{o}^{\star}$ : Grecia-S $25 \mathrm{~km}$ SE Delphi Andikiro 8.5.2005

M. Kadlecová leg. (beide OLML).

Jordanien: 1 \%: Jordan oc. 0-200 m Wadi el Mujib 31 $30^{\circ} \mathrm{N}$ 354'ㄹ 7.-9.4.1994 leg. S. Becvar (OLML).

Kasachstan: 1 ㅇ: Ushch. B. Dalan, $30 \mathrm{~km} \mathrm{z} \mathrm{Alma} \mathrm{Aty,}$ prilovki Kasparyan 6.6.1979;

$2 o^{\top} \sigma^{\top}$ : Ushch. B. Dalan, $28 \mathrm{~km}$ z Alma Aty, prilovki Kasparyan 7.VI.1979, 1 o $^{*}$ : Okr. Alma Ata Uschch. P. Akcai V. Richter 26.5.1977 (alle ZIN).

Kirgistan: 1 o : KIR-Kirghiz. Mts. Ala Archa riv. 6. 1997 $1600 \mathrm{~m}$ coll V. Gurko (OLML).

Türkei: 1 ㅇ: Turkey mer. Cornelek 29.5.1996 $40 \mathrm{~km}$ E of Mut leg. M. Halada. (OLML).

Ukraine: 2 ㅇ ㅇ, $10^{*}$ : Ukraine/ from Z. eupheme pupa (em. Mart 1999), Voikovo/Vil Crimea, M. GascoignePees (NMS, 1 ㅇ Schnee).

Da diese Art von Atanasov (1975) als E. lubricus und von GAUld (1976) als C. tibiale mehr oder weniger ausführlich beschrieben worden ist, wird nachfolgend nur auf Abweichungen und die Variabilität der Art eingegangen.

Variabilität: Körper $12-15 \mathrm{~mm}$, Vorderflügel $6,5-$ 7,5 mm, Fühler 5,5-6,5 mm lang;

Fühler mit 29-36 Gliedern; Occipitalleiste oben hinter Komplexaugen mehrheitlich undeutlich oder erloschen, direkt hinter Ocellen bis auf eine Ausnahme stets vorhanden (Fig. 10); Schläfen im Profil deutlich breiter als die Augen; Discoidella kann in der Mitte des Nervellus, aber auch deutlich unter ihr entspringen; Propodeum mitten längs eingedellt und mit unterschiedlich strukturierter grober Netzrunzelung; Clasper meist wie in Fig. 15 gestaltet, bei einigen wenigen Männchen apico-ventrale Ecke stärker nach hinten reichend als apico-dorsale; Aedoeagus in Lateralsicht apikal stark ausgerandet (Fig. 18a und b), sein dorsal kleinerer und ventral größerer membranöser Lappen mit Mikroglobuli beziehungsweise kurzen Reihen von Mikrotrichae besetzt, sklerotisierter mittlerer Teil zwischen den Membranlappen mit Mikrotubuli (Fig. 18b); bei Mehrzahl der Exemplare Gesicht schwarz mit gelben inneren Orbiten, mitunter Gesicht auch mit gelbem Mittelstreif, seltener äußere Orbiten gelb, roter Scheitelfleck von sehr unterschiedlicher Größe, Petiolus in der Regel bis zu den Spirakeln schwarz, selten ganz rot. Auf zwei variierende Merkmale soll hier noch besonders eingegangen werden:

Der untere Mandibelzahn ist zwar bei allen 33 untersuchten Exemplaren vorhanden, aber er ist viel kleiner als der obere (nur 0,2-mal so lang wie dieser). Es besteht eine Tendenz zu seiner weiteren Reduktion, denn bei zwei Exemplaren ist nur noch eine winzige Ecke von ihm übrig, so dass die Mandibeln bei ihnen de facto einzähnig sind. 
Das bemerkenswerteste variable Merkmal betrifft die Endsporne der Mitteltibien. Bei 10 der Exemplare in coll. SchneE ist nur ein Sporn an jeder Mitteltibia vorhanden. Es fehlt immer der innere kürzere Sporn. Bei drei Tieren weist die eine Tibia einen Sporn und die andere zwei Sporne auf, wobei letztere bei zwei Exemplaren an der rechten und bei einem Exemplar an der linken Tibia vorkommen (Fig. 14a und b). Schließlich besitzen drei weitere Tiere je zwei Endsporne an den Mitteltibien. Auch eines der beiden Männchen aus dem RMNH hat an beiden Mitteltibien zwei Sporne, die links gleich lang und rechts von sehr unterschiedlicher Länge sind. Eines der fünf Exemplare aus dem OLML hat an der linken Mitteltibia zwei Sporne und an der rechten, ebenso wie die übrigen vier an beiden Mitteltibien, nur einen Sporn. Dabei ist diese Variabilität unabhängig vom Geschlecht.

Ob der innere Mitteltibiensporn bei den meisten Tieren gar nicht erst ausgebildet wird oder ob er leicht abbricht, kann vorerst nicht entschieden werden. Er ist meist viel kleiner als der äußere.

Nach ŠEDIVÝ (1970) tritt eine ähnliche Erscheinung auch bei Eucremastus manni (Tschek, 1871) (Cremastinae) auf, so dass SzÉPligeti (1905) die Variante mit zwei Mitteltibienspornen als eigene Art angesehen hat. Atanasov (1975) stellte seine Art lubricus in die Gattung Erigorgus FöRSTER, 1869, obwohl er in seiner Neubeschreibung wichtige nicht zu dieser Gattung passende Merkmale nennt, wie die auch lateral vollständige und scharf am Vorderrand der Mesopleuren endende Praepectalleiste sowie das Vorhandensein von nur einem Endsporn der Mitteltibia. Er erwähnt aber auch, dass bei einem Männchen (vom Fundort Talysh) eine Mitteltibia zwei Endsporne aufweist.

Biologie: Neben dem von Gascoigne-PeEs in der Ukraine ermittelten Wirt Zegris eupheme (EsPER, 1804) sind aus Israel weitere Pieriden-Arten bekannt geworden, aus denen C. lubricum gezüchtet werden konnte (SCHNEE 2014, im Druck).

C. lubricum kommt nach den vorliegenden Daten von Meereshöhe bis $1600 \mathrm{~m}$ vor. Die in Griechenland selbst gefangenen Tiere flogen meist an Wegrändern mit Buschwerk, bestehend aus verschiedenen mediterranen Sträuchern und Rubus-Beständen. Da damals (2007) noch keine Informationen über Wirte vorlagen, wurde den lokal durchaus häufigen blühenden Brassicaceen als Wirtspflanzen potentieller Wirtsraupen keine besondere Beachtung geschenkt.

Verbreitung: Griechenland, Israel, Jordanien, Kasachstan, Kirgistan, Türkei, Ukraine.

\section{Zur verwandtschaftlichen Stellung der Gattung Clypeocampulum}

GAULD (1976) hat zu Recht einen negativen Vergleich von Clypeocampulum mit der nearktischen Gattung Liopterna Townes, 1971 gezogen, die er in seinem Bestimmungsschlüssel für die Gattungen direkt gegenüberstellt. Die von ihm verwendeten Unterscheidungsmerkmale treffen allerdings mehrheitlich nicht zu. Einerseits kannte er weder die Variabilität der Mitteltibiensporne bei C. lubricum noch die beiden neuen Arten. Andererseits konnte er sich nur auf die Beschreibung des (unvollständigen) Holotypus von L. schlingeri durch Townes stützen. Da nach DAsch (1984) weitere Exemplare dieser Art gefunden worden sind, war es möglich, ein Pärchen zu untersuchen. Im Ergebnis dieser Untersuchung sprechen folgende Unterschiede gegen eine nähere Verwandtschaft (Tab. 1).

Tab. 1: Unterschiede zwischen Clypeocampulum und Liopterna

\begin{tabular}{|l|l|}
\hline Clypeocampulum & Liopterna \\
\hline $\begin{array}{l}\text { Praepectalleiste scharf bei } \\
\text { 0.3-0,4 des hinteren Prono- } \\
\text { tumrandes endend }\end{array}$ & $\begin{array}{l}\text { Praepectalleiste nur unten } \\
\text { deutlich, über unterer Pro- } \\
\text { notumecke schwächer }\end{array}$ \\
\hline $\begin{array}{l}\text { Stemmaticum nicht erhöht, } \\
\text { Ocellen nicht eingedrückt }\end{array}$ & $\begin{array}{l}\text { Stemmaticum etwas er- } \\
\text { höht, Ocellen schräg ein- } \\
\text { gedrückt }\end{array}$ \\
\hline $\begin{array}{l}\text { Mesopleuren dicht bis } \\
\text { runzlig punktiert }\end{array}$ & $\begin{array}{l}\text { Mesopleuren glänzend, } \\
\text { wenig punktiert }\end{array}$ \\
\hline $\begin{array}{l}\text { Propodeum höchstens bis } \\
\text { 0,4 der Hintercoxenlänge } \\
\text { reichend }\end{array}$ & $\begin{array}{l}\text { Propodeum bis 0,8 der } \\
\text { Hintercoxenlänge reichend }\end{array}$ \\
\hline $\begin{array}{l}\text { Hintertibienende nicht } \\
\text { verdickt, behaart }\end{array}$ & $\begin{array}{l}\text { Hintertibienende außen } \\
\text { verdickt, glänzend und } \\
\text { unbehaart }\end{array}$ \\
\hline $\begin{array}{l}\text { Hintertarsen länger als die } \\
\text { Hintertibien }\end{array}$ & $\begin{array}{l}\text { Hintertarsen deutlich } \\
\text { kürzer als die Hintertibien }\end{array}$ \\
\hline
\end{tabular}

Da sie einzelne Merkmale teilen, verglich GAULD seine neue Gattung ferner mit den Gattungen Barylypa Förster, 1869 und Habronyx Förster, 1869 sensu GAULD (1976). Er konnte auch unter Berücksichtigung der Flügeladerung, der Morphologie des Thorax und des Aedoeagus zu Recht keine sichere Entscheidung treffen, welcher der bekannten Gattungen sie nahestehen könnte.

Bei der Gattung Camposcopus Förster, 1869 (von GAULD als Untergattung von Habronyx angesehen) entspringt der Subdiscoideus wie bei Clypeocampulum in der Mitte des Postnervulus und die Praepectalleiste erreicht ebenfalls den Vorderrand der Mesopleuren. Sie unterscheidet sich aber deutlich durch zwei vollständig entwickelte Mandibelzähne, einen spitzen Clypeus-Endzahn, eine Querfurche auf dem Pronotum und tiefe Notauli auf der gesamten Mesoscutum-Länge. Bei Barylypa Förster 

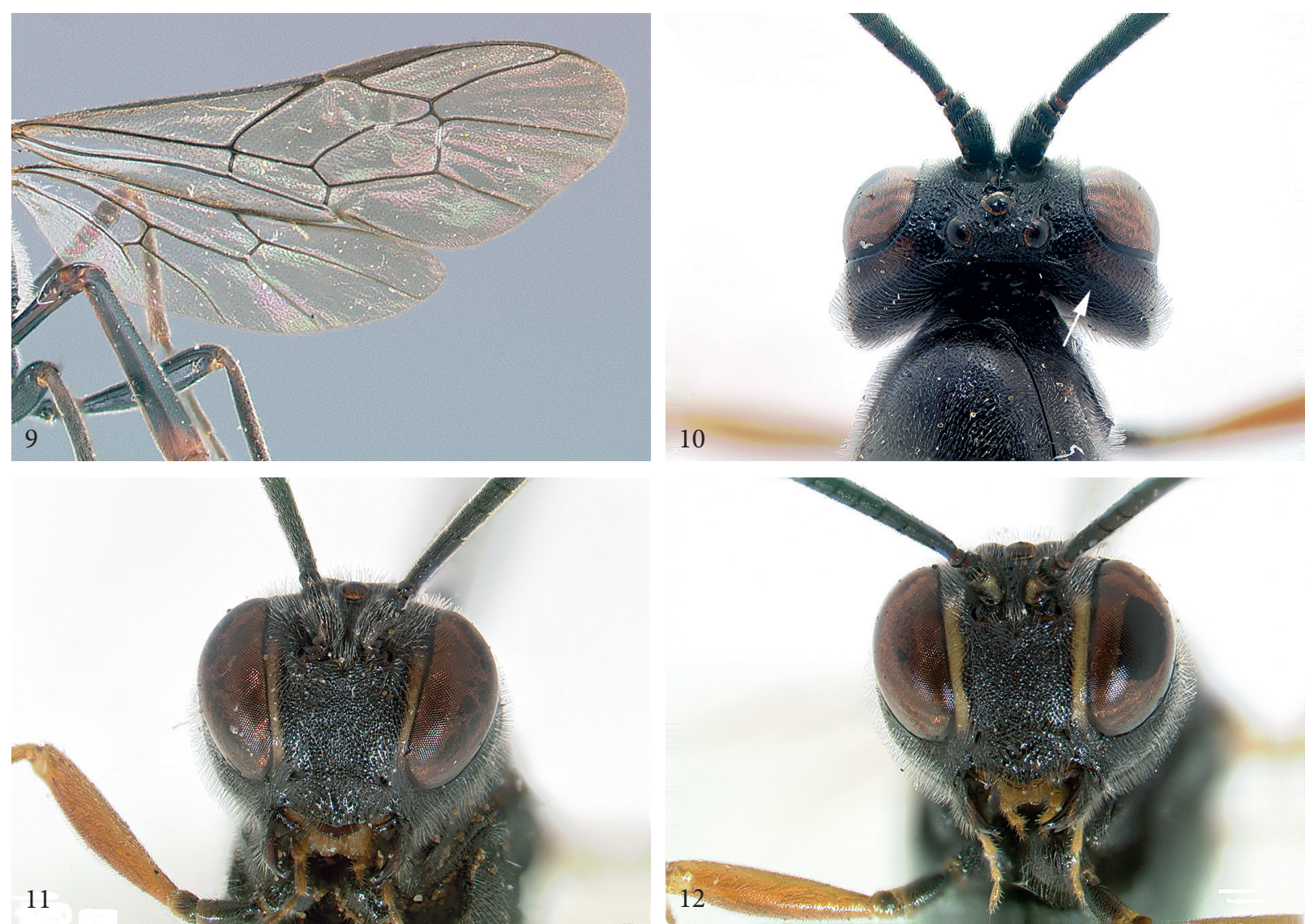

11
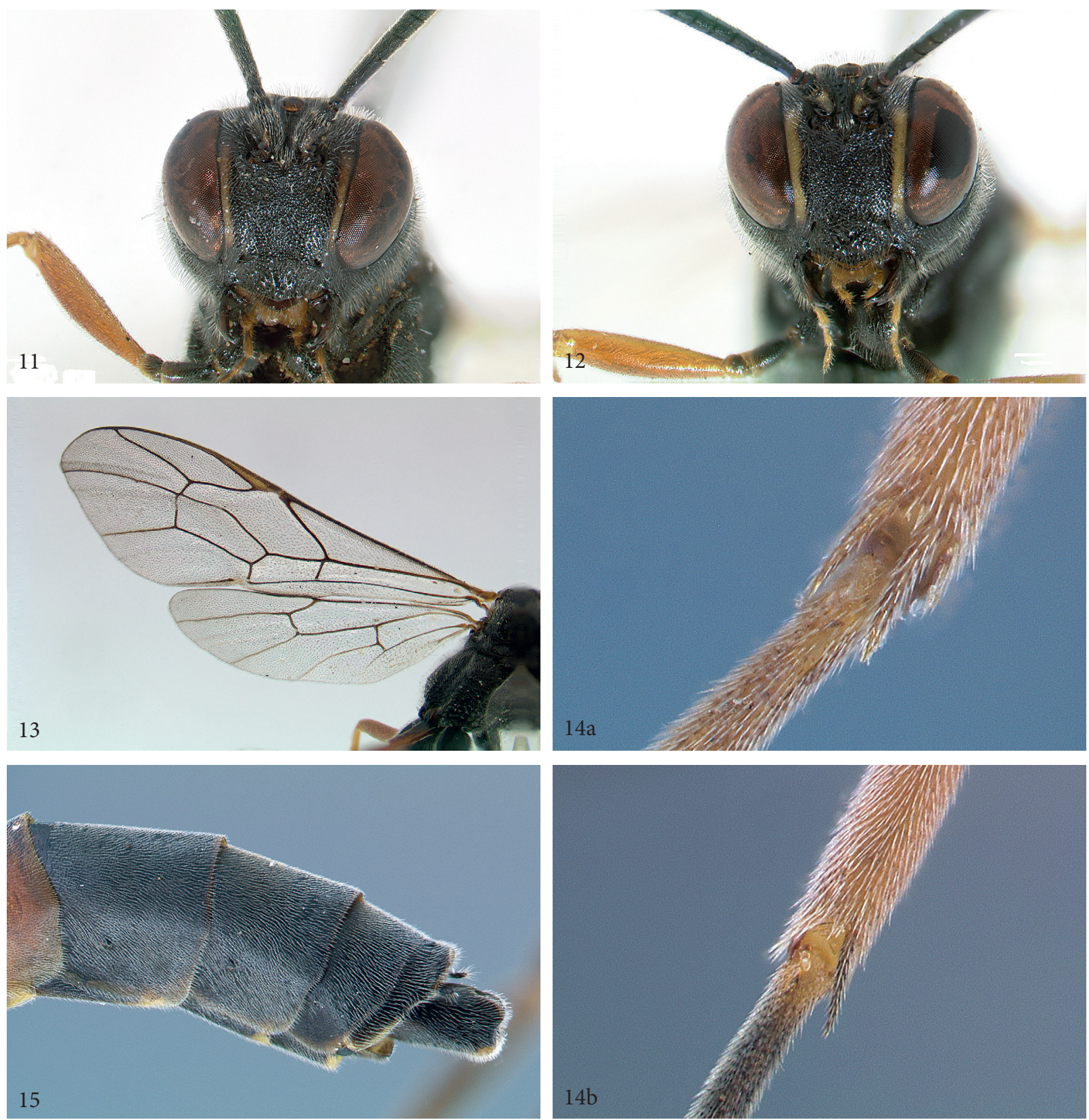

Fig. 9: ㅇ Paratypus Clypeocampulum barbarae sp. n. rechtes Flügelpaar.

Fig. 10-15: Clypeocampulum lubricum (AtanAsov); 10 Kopf und Mesoscutum dorsal ox, 11 und 12 Gesichter von 9 und $\sigma^{\star}, 13$ Vorderflügel $\sigma^{\star}, 14 \mathrm{a}$ Mitteltibia $\sigma^{\star}$ mit 2 Spornen, 14b Mitteltibia $o^{\star}$ mit 1 Sporn, 15 apikale Metasoma-Segmente und Clasper $0^{*}$. 

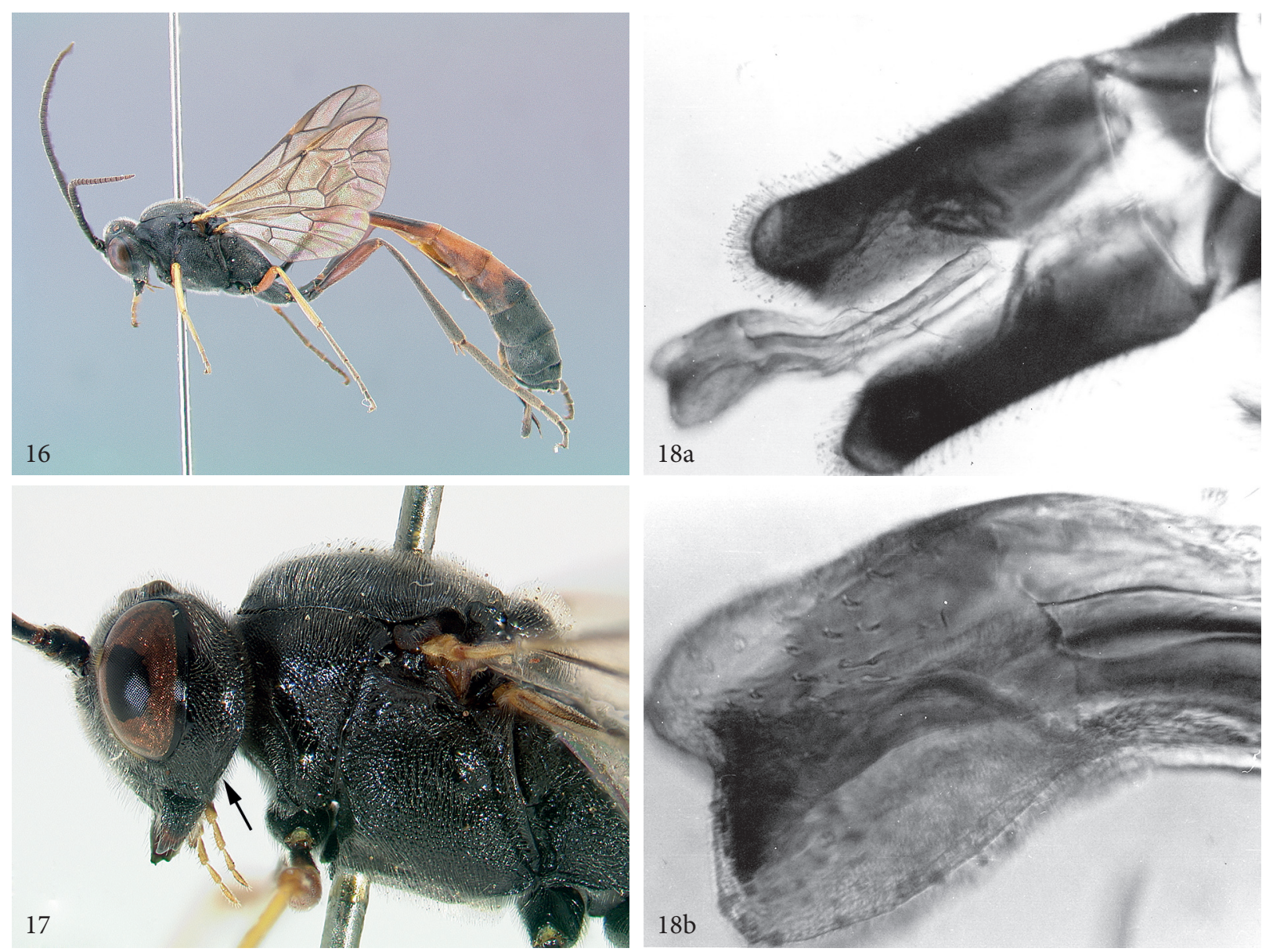

Fig. 16-18b: Clypeocampulum lubricum (AtanAsov); 6 Habitus lateral + , 17 Kopf und Mesosoma lateral ơ, 18a $0^{\star}$ Genitale, $18 \mathrm{~b}$ Aedoeagus.

fehlt wie bei Clypeocampulum eine Querfurche auf dem Pronotum, aber der Subdiscoideus entspringt über der Mitte des Postnervulus, der Endzahn des Clypeus ist vorhanden und die Praepectalleiste reicht kaum über die untere Pronotumecke hinaus, ohne den Vorderrand der Mesopleuren zu erreichen.

Die durch die Auffindung der zwei neuen Arten in der ergänzten Gattungsdiagnose zusammengefassten Merkmale unterstreichen die Eigenständigkeit der Gattung Clypeocampulum innerhalb der Anomaloninae.

Besonders bemerkenswert ist die (fast) vollständige Reduktion des unteren Mandibelzahns bei C. barbarae und C. gauldi sowie der tendenzielle Verlust des zweiten Endsporns der Mitteltibien bei C. lubricum.

Die Untersuchung der Aedoeagi von zwei der hier behandelten Clypeocampulum-Arten hat ergeben, dass ihre Form offenbar für die Gattung charakteristisch ist. Allerdings konnte das Genitale des Holotypus von C. gauldi nicht untersucht werden. Die Aedoeagi der oben zum Vergleich genannten, aber auch aller übrigen bekannten Anomaloninen-Gattungen, die GAULD (1976) abgebildet hat, sind anders geformt.

C. gauldi weicht von den beiden anderen Clypeocampulum-Arten durch den apikal abgerundeten Clypeus, das Vorhandensein von schwachen Notauli und die helle Färbung des Vorderkopfes sowie der Hinterbeine etwas ab. Die Zuordnung zu dieser Gattung ist deshalb nicht völlig zweifelsfrei. Die Gesamtheit der übrigen Merkmale lässt diese Zuordnung aber als gerechtfertigt erscheinen.

Die Gattung ist einerseits westmediterran verbreitet (C. barbarae), andererseits aber auch ostmediterran (C. gauldi und C. lubricum), wobei das Areal von C. lubricum bis nach Mittelasien reicht.

Da eine Weißlingsart auch C. barbarae als Wirt dient, scheint die Gattung Clypeocampulum auf Pieridae spezialisiert zu sein.

\section{Danksagung}

Mein Dank gebührt Dr. C. Van Achterberg (Leiden), Dr. G. Broad und Dr. M. G. Fitton (London), F. Gusenleitner und Dr. M. Schwarz (Linz), Dr. S. Heydon (Davis), Dr. D. R. Kasparyan (St. Petersburg) und Dr. J. Papp (Budapest) für die Ausleihe von Typen und anderen Exemplaren aus den von ihnen betreuten Sammlungen. Prof. Dr. K. Standfuss danke ich herzlich für die Überlassung der von ihm in Griechenland gesammelten Tiere. 
Dr. M. R. Shaw (Edinburgh) verdanke ich die Ausleihe der von C.Stephanescu gezüchteten und der von Gascoigne-Pees erworbenen Exemplare.

Dr. S. M. Blank (Müncheberg) half dankenswerterweise bei der Anfertigung der Fotos am Leica-Fotomikroskop des Senckenberg Deutschen Entomologischen Instituts. A. D. Liston (Müncheberg) bin ich für die Überprüfung der englischen Textstellen dankbar.

\section{Literatur}

AtanAsov, A. Z. 1975: Novyie predstaviteli i opredelitelnaia tablitza palearkticheskih vidov roda Erigorgus (Hymenoptera, Ichneumonidae). - Zoologicheski Journal 54: 1480-1487.

DASCH, C. E. 1984: Ichneumon-flies of America North of Mexico: 9. Subfamilies Theriinae and Anomaloninae. - Memoirs of the American Entomological Institute 36: $610 \mathrm{pp}$.
GAUld, I. D. 1976: The classification of the Anomaloninae (Hymenoptera: Ichneumonidae). - Bulletin of the British Museum (Natural History) Entomology 33: $1-135$.

Schnee, H. 2014: Anomaloninae aus Israel und Palästina. - Entomologische Zeitschrift 124, im Druck.

ŠEDIVÝ. J. 1970: Westpaläarktische Arten der Gattungen Dimorpha, Pristomerus, Eucremastus und Cremastus (Hym. Ichneumonidae). - Acta scientiarum naturalium Brno 4 (11): 1-38.

SzÉPligeti, G. 1905: Hymenoptera. Ichneumonidae (Gruppe Ophionoidea), Subfam. Pharsaliinae Porizontinae. - Genera Insectorum, Bruxelles 34: 1-68.

Townes, H. 1971: The genera of Ichneumonidae, part 4. - Memoirs of the American Entomological Institute 17, Ann Arbor: 372 pp. 